\title{
Cingulate gyrus epilepsy: semiology, invasive EEG, and surgical approaches
}

\author{
Chien-Chen Chou, MD, ${ }^{1,3,5}$ Cheng-Chia Lee, MD, PhD, ${ }^{1,2,5}$ Chun-Fu Lin, MD, ${ }^{1,2}$ Yi-Hsiu Chen, BS, ${ }^{2}$ \\ Syu-Jyun Peng, PhD, ${ }^{4}$ Fu-Jung Hsiao, PhD, ${ }^{5}$ Hsiang-Yu Yu, MD, ${ }^{1,3,5}$ Chien Chen, MD, ${ }^{1,3}$ \\ Hsin-Hung Chen, MD, ${ }^{1,2}$ and Yang-Hsin Shih, MD ${ }^{1,2}$
}

${ }^{1}$ School of Medicine and ${ }^{5}$ Brain Research Center, National Yang-Ming University; Departments of ${ }^{2}$ Neurosurgery and ${ }^{3}$ Neurology, Neurological Institute, Taipei Veterans General Hospital; and ${ }^{4}$ Professional Master Program in Artificial Intelligence in Medicine, College of Medicine, Taipei Medical University, Taipei, Taiwan

OBJECTIVE The semiology of cingulate gyrus epilepsy is varied and may involve the paracentral area, the adjacent limbic system, and/or the orbitofrontal gyrus. Invasive electroencephalography (iEEG) recording is usually required for patients with deeply located epileptogenic foci. This paper reports on the authors' experiences in the diagnosis and surgical treatment of patients with focal epilepsy originating in the cingulate gyrus.

METHODS Eighteen patients (median age 24 years, range 5-53 years) with a mean seizure history of 23 years (range 2-32 years) were analyzed retrospectively. The results of presurgical evaluation, surgical strategy, and postoperative pathology are reported, as well as follow-up concerning functional morbidity and seizures (median follow-up 7 years, range 2-12 years).

RESULTS Patients with cingulate gyrus epilepsy presented with a variety of semiologies and scalp EEG patterns. Prior to ictal onset, $11(61 \%)$ of the patients presented with aura. Initial ictal symptoms included limb posturing in $12(67 \%)$, vocalization in 5 , and hypermotor movement in 4 . In most patients $(n=16,89 \%)$, ictal EEG presented as widespread patterns with bilateral hemispheric origin, as well as muscle artifacts obscuring the onset of EEG during the ictal period in 11 patients. Among the 18 patients who underwent resection, the pathology revealed mild malformation of cortical development in 2, focal cortical dysplasia (FCD) lb in 4, FCD Ila in 4, FCD Ilb in 4, astrocytoma in 1, ganglioglioma in 1, and gliosis in 2. The seizure outcome after surgery was satisfactory: Engel class IA in 12 patients, IIB in 3, IIIA in 1, IIIB in 1, and IVB in 1 at the 2-year follow-up.

CONCLUSIONS In this study, the authors exploited the improved access to the cingulate epileptogenic network made possible by the use of 3D electrodes implanted using stereoelectroencephalography methodology. Under iEEG recording and intraoperative neuromonitoring, epilepsy surgery on lesions in the cingulate gyrus can result in good outcomes in terms of seizure recurrence and the incidence of postoperative permanent deficits.

https://thejns.org/doi/abs/10.3171/2020.1.FOCUS19914

KEYWORDS cingulate gyrus; limbic system; epileptogenic networks; topology; epilepsy surgery; invasive electroencephalography; iEEG; stereoelectroencephalography; SEEG; subdural grid

$\mathrm{T}$ HE semiology and electroencephalography (EEG) patterns of cingulate gyrus epilepsy are difficult to characterize. Clinical findings could be misleading in predicting the localization of seizure onset. ${ }^{1,13}$ The variations in semiological features can be attributed to the unique cytoarchitecture and neural fiber projections in the various regions of the cingulate gyrus. ${ }^{6}$ There are several methods for the division of the cingulate gyrus; $;, 21$ however, the 4-region neurobiological model of the cingulate cortex is the most useful because it is based on observations pertaining to circuitry as well as structural and functional imaging studies..$^{21-26}$ The cingulate cortex includes the anterior cingulate cortex (ACC), middle cingulate cortex (MCC), posterior cingulate cortex (PCC),

ABBREVIATIONS ACC = anterior cingulate cortex; $A E D=$ antiepileptic drug; $E E G=$ electroencephalography; FCD = focal cortical dysplasia; $H F O=$ high-frequency oscillation; iEEG = invasive EEG; IONM = intraoperative neuromonitoring; IPL = inferior parietal lobule; $M C C=$ middle cingulate cortex; $\mathrm{mMCD}=$ mild malformation of cortical development; PCC = posterior cingulate cortex; RSC = retrosplenial cortex; SDG = subdural grid; SEEG = stereo-EEG; SMA = supplementary motor area.

SUBMITTED November 27, 2019. ACCEPTED January 27, 2020.

INCLUDE WHEN CITING DOI: 10.3171/2020.1.FOCUS19914. 
and retrosplenial cortex (RSC), all of which can be further divided into subregions. Based on cytoarchitectural analysis, afferent connections, and primate functional studies, the semiological features of seizures can be separated into several groups. ${ }^{7}$ The groups can be organized along a rostrocaudal axis, representing bands within a spectrum rather than rigid categories. Anterior seizure organization is more likely to present integrated behavior during seizures. Distal stereotypies are associated with the most anterior prefrontal localizations, whereas proximal stereotypies are associated with posterior prefrontal regions. ${ }^{7}$

Semiologies from the posterior cingulate gyrus are even harder to differentiate; ${ }^{9}$ they include bilateral asymmetrical tonic seizure, hypermotor seizure, dialeptic seizure, and automotor seizure. The ictal activities of bilateral asymmetrical tonic seizure and hypermotor seizure originating in the PCC generally spread to frontal (lateral premotor area, orbitofrontal cortex, supplementary motor area [SMA], and ACC) and parietal (precuneus, posterior cingulate gyrus, inferior parietal lobule [IPL], and postcentral gyrus) areas. The seizures associated with dialeptic seizure and automotor seizures generally spread to medial temporal or IPL areas. Again, the abundance of fiber projections and connections increases the variety of semiological features.

Invasive EEG (iEEG) is widely used to explore the epileptogenic focus in the cingulate cortex via subdural grid (SDG) implantation or stereo-EEG (SEEG) recording. In this paper, we explored the difficulties in diagnosing cingulate gyrus epilepsy and summarized the surgical results and complications associated with the management of cingulate epilepsies.

\section{Methods}

\section{Patients and iEEG Acquisition}

A total of 18 patients (median age 24 years, range 5-53 years) with cingulate gyrus epilepsy and a median seizure history of 23 years (range 2-32 years) were retrospectively analyzed. Data pertaining to functional morbidity and seizures were collected from presurgical evaluations, diagnostics, surgical strategy, and postoperative follow-up (median follow-up 7 years, range 2-12 years).

Among the patients in this study, 8 presented with potentially epileptogenic lesions on preoperative MRI, and 10 others were MRI-negative in a phase I study. In 5 of the cases presenting clearly defined MRI lesions with compatible semiology and scalp EEG, the surgeon proceeded directly to resection of the potentially epileptogenic zone. For patients who were MRI-negative and/or presented discordance between the semiology and scalp EEG findings, physicians opted for iEEG (phase II study) to improve localization and lateralization. Our facility in Taipei provides 2 types of iEEG: SDG/strip electrode implantation, and SEEG.

SDGs or strips (Ad-Tech Medical Instrument Co.) were custom-made for 5 patients based on seizure semiology and data obtained from chronic video-EEG monitoring, neuropsychological tests, MRI, PET, and magnetoencephalography (MEG). The grids or strips ranged in size from 40 to 117 platinum electrodes (median 95 electrodes). In cases with cingulate gyrus epilepsy, unilateral or bilateral SDGs/strip electrodes with and/or without depth electrodes were used to capture ictal EEG readings from the lateral cortex, mesial cortex, or bottom-of-sulcus. In the placement of grid/strip electrodes for common cingulate gyrus epilepsy, a bicoronal craniotomy was preferred, wherein the bone flap was removed and a unilateral and/ or bilateral U-shaped dural opening was made based on the sagittal sinus. After the interhemispheric space was exposed, SDG/strip electrodes were placed on the lateral as well as mesial cortical surfaces. In some cases, depth electrodes were also used to record EEG signals directly from the cerebral parenchyma (Fig. 1A). Subdural EEG readings (sampled at $1024 \mathrm{~Hz}$ ) were recorded on a Nicolet system (Natus Medical Inc.) with 8-48 contacts per SDG/ strip electrode and a maximum number of 100 recording channels. Data were digitized to hard disk at 16-bit resolution. Subdural EEG readings were recorded for a period of 5-7 days under video camera surveillance to validate the electroclinical features of the seizures.

SEEG was collected from 9 patients with drug-resistant cingulate gyrus epilepsy (Fig. 1B and C). All of the patients underwent surgical implantation (via stereotaxy) of intracranial depth electrodes (ADtech, 6-16 contacts: multicontacts, interval 3-10 $\mathrm{mm}$, diameter $0.86 \mathrm{~mm}$ ). The procedures were conducted in accordance with the methodology introduced at Saint Anne Hospital (Paris, France) in 1974. ${ }^{8,17}$ The trajectory, location, and number of the implanted electrodes depended on clinical information and the results of phase I studies, as mentioned above. SEEG readings (sampled at $1024 \mathrm{~Hz}$ ) were recorded on a Nicolet system (Natus Medical Inc.) with 8-10 contacts per intracranial electrode and a maximum number of 100 recording channels. Data were digitized to hard disk at 16-bit resolution. SEEG readings were recorded for 7-14 days under video camera surveillance to validate the electroclinical features of the seizures.

One female patient (case 14) underwent SDG recording as well as SEEG due to the failure of the first SDG-tailored resection. That patient had only partial control of seizures following lesionectomy via craniotomy. SEEG recordings of deep EEG signals obtained 5 years later revealed that the seizure onset zone was located in the posterior cingulate gyrus, which was invisible to high-resolution MRI observation.

\section{Reconstruction of Subdural/Depth Electrodes and Pial Surface}

The positions of the implanted subdural/depth electrodes were determined postoperatively via high-resolution CT scans and structural MRI without contrast. FreeSurfer was used to reconstruct a 3D model of the cortical surface using preoperative high-resolution MRI. Postoperative CT was coregistered to the preoperative MRI of the same patient using normalized mutual information for a registration algorithm included in a commercial image analysis software package (ANALYZE version 12.0, Biomedical Imaging Resource). All contact coordinates were obtained from registered postoperative CT scans using ANALYZE software and embedded in the reconstructed 3D model of the cortex. 

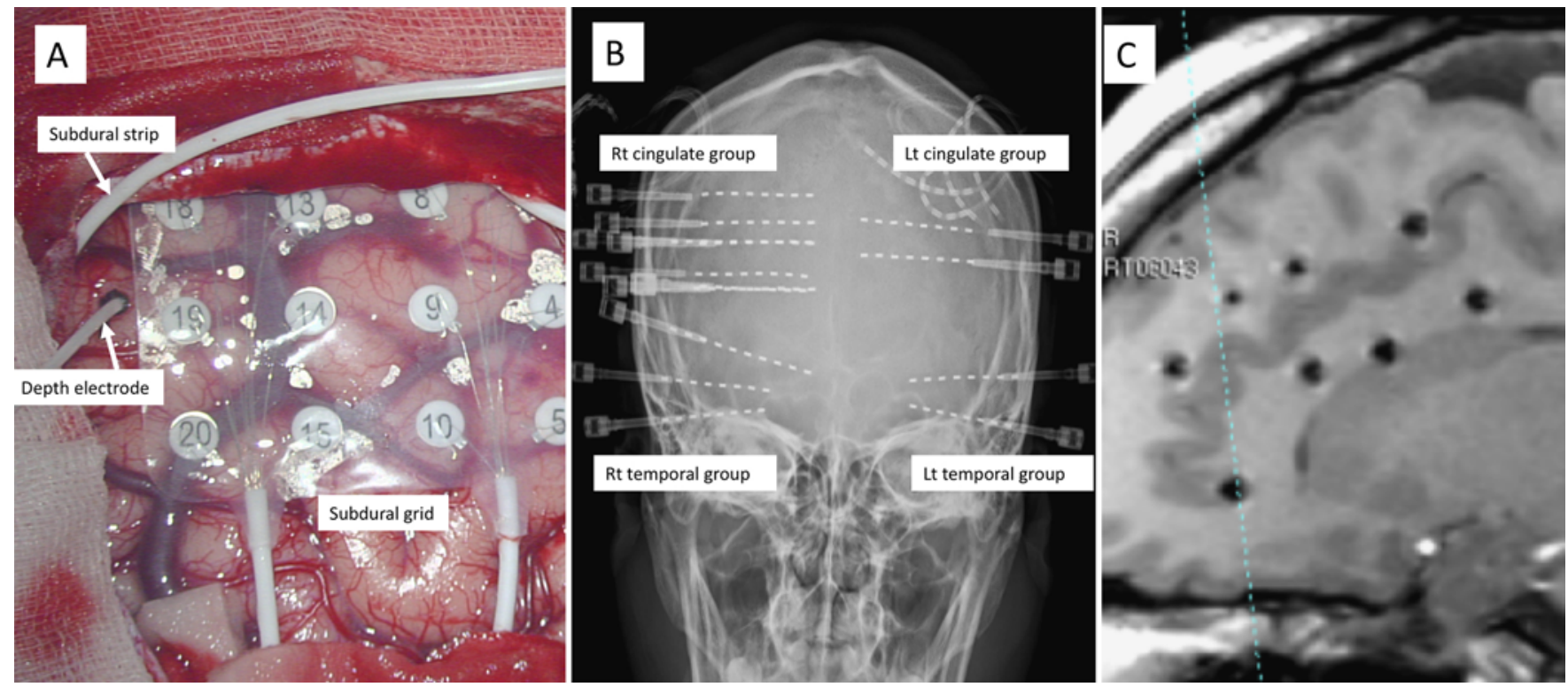

FIG. 1. Invasive EEG recording from the cingulate gyrus. SDG/strip implantation combined with one depth electrode (A), and SEEG via multiple depth electrode implantations (B). The MR image shows the contacts of depth electrodes around the cingulate sulcus $(\mathbf{C})$.

\section{Construction of High-Frequency Oscillation Networks Based on iEEG Recordings}

The construction of high-frequency oscillation (HFO) networks from iEEG involved the following outlined below. ${ }^{11}$ First, we pruned the iEEG segments of each seizure (from 60 seconds prior to seizure onset until 30 seconds after seizure termination). A bipolar montage obtained by subtracting iEEG monopolar signals recorded from two adjacent contacts was used to limit the influence of the common reference. Second, we bandpass-filtered the bipolar iEEG (third-order Butterworth, zero-phase digital filtering) in the HFO band of $80-250 \mathrm{~Hz}$ and notch-filtered (third-order Butterworth, zero-phase digital filtering) the data at 120, 180, and $240 \mathrm{~Hz}$. Third, we divided the iEEG into 1-second windows with 0.5 -second overlaps and normalized the data from each electrode within the 1-second window to obtain zero mean and unit variance. Fourth, we applied cross-correlation to the preprocessed iEEG between the two electrodes and selected the maximum correlation within time delays of \pm 0.5 seconds. Fifth, we determined the statistical significance of the correlation value using an analytical procedure that interprets our choice of an extremum. ${ }^{11}$ Sixth, we corrected for multiple significance tests using a linear step-up procedure, while controlling the false detection rate with $p=0.05$. The interelectrode cross-coefficient was measured between all pairs of electrodes for each 1-second window.

\section{Results}

\section{Semiology and Scalp EEG Findings}

Table 1 summarizes the various semiologies and scalp EEG presentations in patients with cingulate gyrus epilepsy. Patients in this group were drug-resistant, with the result that they were taking between 2 and 7 antiepileptic drugs (AEDs). Prior to ictal onset, 7 of the patients had no aura, 1 patient had vertigo or dizziness, 1 patient reported déjà vu, 2 patients had anxiety and fear, and 7 patients had aura but the specifics were indescribable. Initial ictal symptoms included limb posturing in 12 , vocalization in 5 , hypermotor movement of the limbs in 4 , versive head movement in 4 , oral/limbs automatism in 3, elementary/ myoclonic movement in 2 , and ictal pouting in 1 . Ictal EEG patterns in most of the patients $(\mathrm{n}=16)$ were widespread and originated in the bilateral hemispheres. Muscle artifacts obscured the onset of EEG during the ictal period in 11 of the patients.

\section{Invasive EEG Findings}

The electroclinical features of the seizures in $13 \mathrm{pa}-$ tients who presented with drug-resistant cingulate gyrus epilepsy were validated by simultaneously recording video and iEEG signals for an average of 9 days (range 7-14 days). Data from a total of 97 seizures were collected. Sixty-seven seizures (69\%) were focal onset with impairment of awareness, and 30 seizures (31\%) presented focal to bilateral hemisphere involvement.

The 13 patients were assessed using a median of 137 electrode contacts (range 54-158 contacts), and all of the contacts were analyzed individually. Three-dimensional maps were reconstructed for all of those patients, including the positions of SDG/depth electrodes. Specifically, the electrodes were mapped within brain images extracted from high-resolution structural images of each patient. It was observed that the HFO network changed constantly throughout the evolution of the seizures. The early electrodecremental events involving HFO between 80 and 250 $\mathrm{Hz}$ (so-called "ripples") were associated not only with the epileptogenic zone but also bilaterally with the head and body of the hippocampus. When combined with the vari- 
TABLE 1. Semiological and electrophysiological data from patients with cingulate gyrus epilepsy

\begin{tabular}{|c|c|c|c|c|c|c|c|c|}
\hline $\begin{array}{l}\text { Pt } \\
\text { No. }\end{array}$ & $\begin{array}{c}\text { Age (yrs) } \\
\text { Sex }\end{array}$ & Side & $\begin{array}{l}\text { No. of } \\
\text { AEDs }\end{array}$ & $\begin{array}{c}\text { Lesion } \\
\text { (MRI) }\end{array}$ & Location & Aura* $^{*}$ & Main Semiological Elements & Scalp EEG Findings \\
\hline 1 & $15, F$ & Rt & 2 & No & MCC & 4 & Lt arm \& leg posturing & $\mathrm{F} 4>\mathrm{F} 3$ \\
\hline 2 & $13, M$ & Rt & 2 & No & MCC & 5 & Tonic posturing It leg & F8, T4 \\
\hline 3 & $11, F$ & $\mathrm{Lt}$ & 2 & Yes & ACC & 5 & Staring, eye blinking, finger automatism, smacking & F3, F7, T3 \\
\hline 4 & $40, F$ & Rt & 3 & No & ACC & 1 & Rt versive, hypermotor & Fp2, F4, C4 \\
\hline 5 & $21, F$ & Rt & 2 & Yes & MCC & 4 & Tonic posturing It shoulder, arm & $\mathrm{F} 4, \mathrm{C} 4, \mathrm{P} 4$ \\
\hline 6 & $53, \mathrm{M}$ & Rt & 2 & Yes & MCC & 5 & Lt arm posturing & $\mathrm{F} 4>\mathrm{F} 3, \mathrm{w} /$ muscle artifact \\
\hline 7 & $35, F$ & $\mathrm{Lt}$ & 3 & Yes & ACC & 4 & Rt arm posturing, hypermotor & $\mathrm{F} 3>\mathrm{F} 4, \mathrm{w} /$ muscle artifact \\
\hline 8 & $14, F$ & $\mathrm{Lt}$ & 3 & Yes & ACC & 5 & Vocalization, rt versive, bilat arm tonic posturing & $\mathrm{F} 3>\mathrm{F} 4, \mathrm{w} /$ muscle artifact \\
\hline 9 & $28, F$ & $\mathrm{Lt}$ & 3 & No & ACC & 4 & Myoclonic rt hand & $\mathrm{F} 3>\mathrm{F} 4, \mathrm{w} /$ muscle artifact \\
\hline 10 & $27, \mathrm{M}$ & $\mathrm{Lt}$ & 3 & Yes & MCC & 5 & Vocalization, It tonic & $\mathrm{F} 3>\mathrm{F} 4, \mathrm{w} /$ muscle artifact \\
\hline 11 & $19, \mathrm{~F}$ & Rt & 3 & No & ACC & 3 & Lt arm posturing, hypermotor & $\mathrm{F} 3, \mathrm{~F} 4, \mathrm{C} 3, \mathrm{C} 4, \mathrm{w} /$ muscle artifact \\
\hline 12 & $38, F$ & $\mathrm{Lt}$ & 7 & Yes & RSC & 4 & Tonic posturing, bilat arm & P4-T6 \\
\hline 13 & $18, M$ & $\mathrm{Lt}$ & 2 & No & ACC & 4 & Shouting, tonic posturing, upper gazing & $\mathrm{F} 3>\mathrm{F} 4, \mathrm{w} /$ muscle artifact \\
\hline 14 & $29, \mathrm{~F}$ & $\mathrm{Lt}$ & 4 & No & RSC & 5 & Chewing (oral automatism), versive, tonic posturing & $\mathrm{C} 3, \mathrm{P} 1, \mathrm{O} 1$ \\
\hline 15 & $12, \mathrm{M}$ & Rt & 4 & No & ACC & 2 & $\begin{array}{l}\text { 4-limb extension (tonic posturing), upward gazing, } \\
\text { head versive to It, motionless staring }\end{array}$ & T4, C3, F3, w/ muscle artifact \\
\hline 16 & $5, \mathrm{M}$ & $\mathrm{Lt}$ & 4 & No & PCC & 4 & Elementary movement of rt limbs & $\mathrm{C} 3>\mathrm{C} 4, \mathrm{w} /$ muscle artifact \\
\hline 17 & $37, F$ & $\mathrm{Lt}$ & 5 & Yes & ACC & 3 & Vocalization, hypermotor & $\mathrm{F} 3>\mathrm{F} 4, \mathrm{w} /$ muscle artifact \\
\hline 18 & $39, \mathrm{M}$ & Rt & 5 & No & ACC & 5 & $\begin{array}{l}\text { Saliva drooling (oral automatism), vocalization, ictal } \\
\text { pouting, fixed upward gaze, tonic posture }\end{array}$ & $\mathrm{F} 4>\mathrm{F} 3, \mathrm{w} /$ muscle artifact \\
\hline
\end{tabular}

$\mathrm{Pt}=$ patient.

${ }^{*}$ Aura categories: 1 = vertigo/dizziness; 2 = déjà vu; $3=$ anxiety/fear; $4=$ indescribable; $5=$ none.

ous frequencies associated with the cingulate gyrus, these findings revealed rapid changes in the frequency content (i.e., "chirps"). This may explain the strong relationship between the cingulate gyrus and the hippocampus (Fig. 2, Video 1).

VIDEO 1. Clip showing connectivity between the cingulate gyrus and related limbic system during onset of typical cingulate gyrus seizure. The video showed the connectivity change from the initiation of ictal onset, to the early stage of ictal onset, to the middle stage of ictal onset, to the late stage of ictal onset. Copyright Cheng-Chia Lee. Published with permission. Click here to view.

\section{Surgical Outcomes and Pathological Diagnosis}

Figure 3 presents the locations of resection, and Table 2 presents the surgical outcomes and pathological identities. The pathology of the 5 MRI-visible lesions that did not undergo iEEG analysis revealed focal cortical dysplasia (FCD) IIa in 2 patients, FCD IIb in 2, and gliosis in 1 (case 4). Among the 13 patients who underwent iEEG analysis, the origin of ictal onset was identified only in the electrodes surrounding the lesion, and the pathology revealed mild malformation of cortical development (mMCD) in 2 patients, FCD Ib in 4, FCD IIa in 2, FCD IIb in 2, astrocytoma in 1, ganglioglioma in 1, and gliosis in 1. All of the patients were followed for more than 2 years until the end of the study. Seizure outcomes after surgery were satisfactory: Engel class IA in 12 patients, IIB in 3, IIIA in 1 , IIIB in 1, and IVB in 1. Intraoperative neuromonitoring (IONM) was used during resection on 8 patients with cingulate gyrus epilepsy. After resection, SMA syndrome was identified in 5 patients and all cases resolved spontaneously within 2 weeks.

Notably, we had 1 case with astrocytoma who underwent SEEG (case 6), and 1 case with ganglioglioma who underwent SDG implantation (case 3). We did not routinely perform iEEG studies for lesional epilepsy, especially tumor cases. However, the lesion in the astrocytoma case was located in the MCC, which is an eloquent area for motor and/or sensory function. Therefore, we applied the SEEG for functional mapping (mainly) and epileptogenic lesion definition. For the case with ganglioglioma, we applied SDG recording to confirm the seizure onset because of discordant results from MRI (orbitofrontal region) and clinical EEG (temporal region).

\section{Discussion}

\section{Characteristics of Semiology in Different Subregions of Cingulate Gyrus Epilepsy}

The ACC plays an important role in cognitive control. ${ }^{12,18}$ However, in both our study and in previous results, the main seizure semiologies from ACC were fear, vocalization, hypermotor, and pouting, which were more related to nearby frontal cortex but not the ACC itself. ${ }^{2,5,15}$ In the $\mathrm{MCC}$, there were only few cases reporting the initial ictal symptoms, including jigging or painful sensation of the head, and stiffness of the body or limbs. ${ }^{14,28}$ Among 5 cases of epilepsy originating from the MCC in the current study, the characteristic semiology was posturing of the limbs, but not sensory symptoms noted in previous stud- 

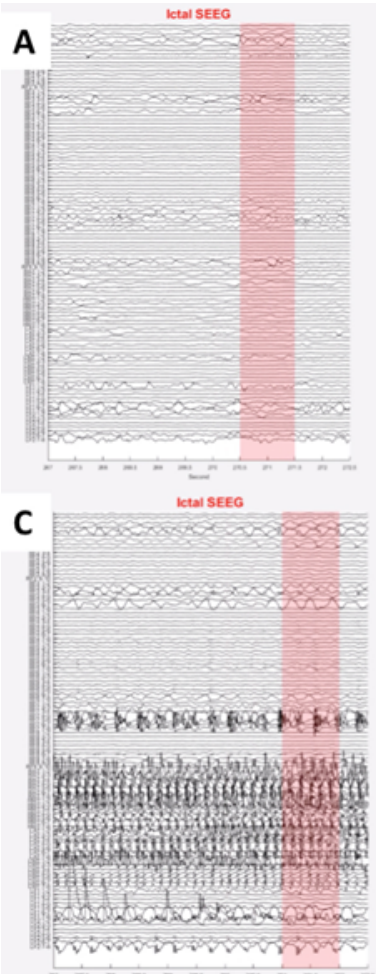
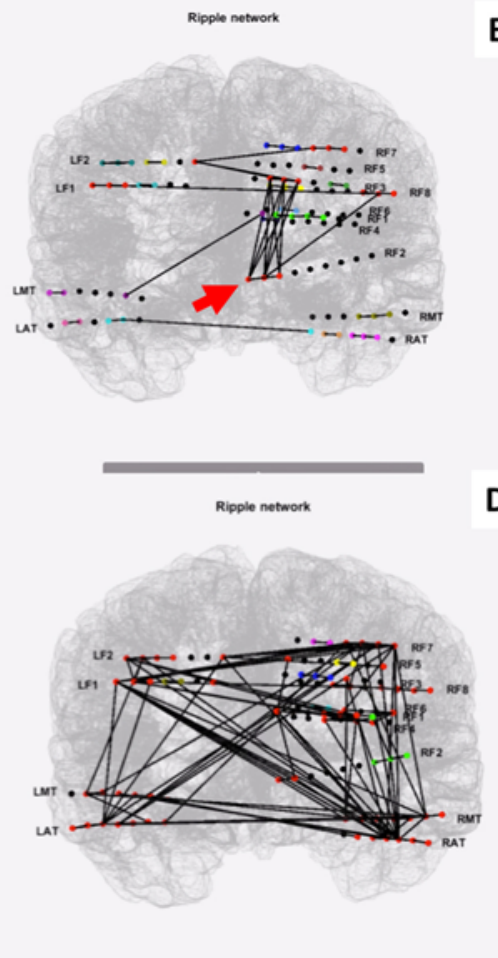
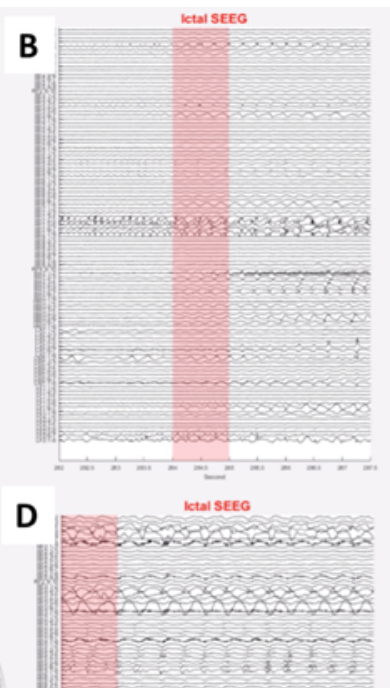

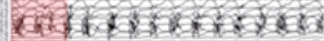

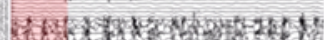
Hom (II) मोin

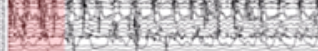

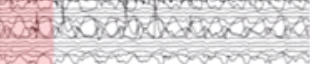

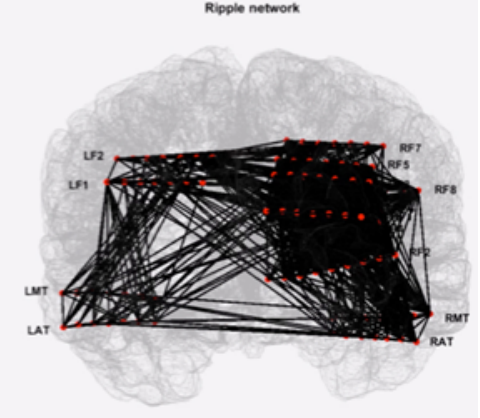

FIG. 2. Connectivity between the cingulate gyrus and related limbic system during onset of typical cingulate gyrus seizure: initiation of ictal onset (A), early stage of ictal onset (B), middle stage of ictal onset (C), and late stage of ictal onset (D; also see Video 1). The inner electrode contacts of RF2, RF3, and RF4 were located within the ACC, which was the seizure onset zone (arrow) in this demonstrative case. Instead of electrode contacts in the ACC, the inner electrode contacts of RMT and RAT were located within the hippocampus, which was irritated from the early to late stages of the ictal period.
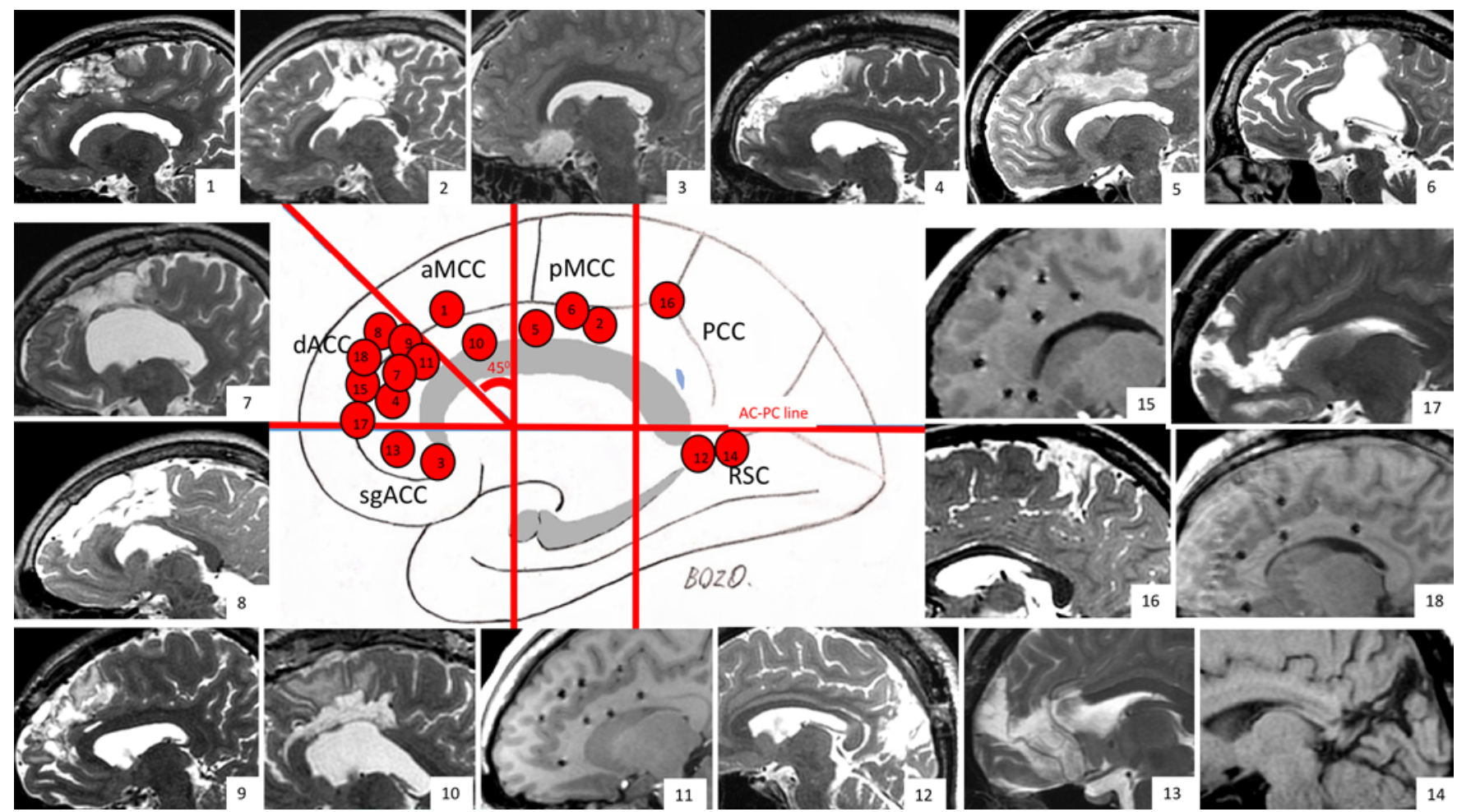

$B 020$.
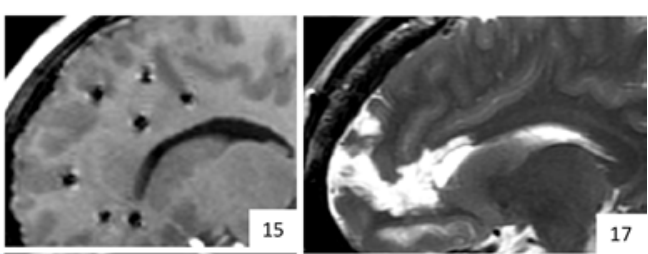

FIG. 3. Location of cingulate gyrus lesionectomy in all 18 patients and standardization of the lesion into the 4-region neurobiological model of the cingulate cortex. $\mathrm{AC}-\mathrm{PC}=$ anterior commissure-posterior commissure; aMCC = anterior MCC; $\mathrm{dACC}=$ dorsal ACC; pMCC = posterior MCC; $\mathrm{sgACC}=$ subgenual ACC. 
TABLE 2. Invasive EEG study and surgical results for patients with cingulate gyrus epilepsy

\begin{tabular}{|c|c|c|c|c|c|c|c|}
\hline Pt No. & Location & iEEG & Causes of Adjacent Gyrus Resection & Outcome* & Pathology & SMA Syndrome & IONM for Surgery \\
\hline 1 & MCC & SDG & Adjacent gyrus is also seizure onset zone & IA & FCD lb & No & No \\
\hline 2 & MCC & SDG & For surgical approach purpose & IA & FCD lla & Yes & Yes \\
\hline 3 & ACC & SDG & No adjacent gyrus resection & IA & Ganglioglioma & No & No \\
\hline 4 & ACC & None & Adjacent gyrus is also seizure onset zone & IIB & Gliosis & Yes & Yes \\
\hline 5 & MCC & None & No adjacent gyrus resection & IA & FCD lla & No & No \\
\hline 6 & MCC & SEEG & Adjacent gyrus is also seizure onset zone & IA & Astrocytoma & Yes & Yes \\
\hline 7 & ACC & SEEG & For surgical approach purpose & IIB & FCD lb & No & No \\
\hline 8 & ACC & None & Adjacent gyrus is also seizure onset zone & IA & FCD Ilb & No & Yes \\
\hline 9 & ACC & SDG & Adjacent gyrus is also seizure onset zone & IVB & Gliosis & No & Yes \\
\hline 10 & MCC & SEEG & No adjacent gyrus resection & IA & FCD IIb & Yes & No \\
\hline 11 & ACC & SEEG & Adjacent gyrus is also seizure onset zone & IA & FCD IIb & No & No \\
\hline 12 & RSC & None & Adjacent gyrus is also seizure onset zone & IIIB & FCD IIb & No & Yes \\
\hline 13 & ACC & SEEG & For surgical approach purpose & IA & FCD Ila & No & Yes \\
\hline 14 & RSC & SDG + SEEG & Adjacent gyrus is also seizure onset zone & IA & mMCD, type I \& II & No & No \\
\hline 15 & ACC & SEEG & Adjacent gyrus is also seizure onset zone & IIIA & mMCD, type I \& II & No & No \\
\hline 16 & PCC & SEEG & Adjacent gyrus is also seizure onset zone & IA & $\mathrm{FCD} \mathrm{lb}$ & Yes & Yes \\
\hline 17 & ACC & None & For surgical approach purpose & IA & FCD Ila & No & No \\
\hline 18 & ACC & SEEG & Adjacent gyrus is also seizure onset zone & $\| B$ & FCD lb & No & No \\
\hline
\end{tabular}

* Seizure outcomes were evaluated by Engel's classification.

ies. In this study, we only collected 3 cases of epilepsy from the posterior part of the cingulate gyrus (including the PCC and RSC). Their semiology included elementary motor symptoms in 2 patients and automatism in 1 patient. Our results were compatible with previous findings of epilepsy in the PCC, i.e., either prominent motor symptoms as frontal lobe epilepsy or dyscognitive symptoms as temporal lobe epilepsy $\mathrm{y}^{2,9}$

\section{Using iEEG to Examine Potential Epileptogenic Zones in Cases of Cingulate Gyrus Epilepsy}

The standard technique used to delineate the ictal onset zone in cases of refractory neocortical epilepsies involves obtaining chronic extraoperative iEEG recordings using SDG/strip electrodes. ${ }^{16}$ The ability to directly observe the surface of the brain facilitates the placement of subdural electrodes, thereby making it possible to obtain excellent electroencephalographic signals related to the localization of seizure onset and eloquent areas of the cortex. Nonetheless, craniotomy and subsequent monitoring are invasive neurosurgical procedures with the potential for neurological and medical morbidity. This potential has prompted a number of epileptologists and neurosurgeons to employ stereotaxy in the placement of depth electrodes aimed at identifying the epileptogenic zone, seizure onset zone, irritative zone, and even symptomatic zone. In the last decade, physicians have increasingly opted for use of SEEG electrodes to identify the epileptogenic zone in patients with cingulate gyrus epilepsy. Since its advent in Europe, SEEG has gradually gained acceptance in North America and Asia. Deep epileptogenic lesions, such as those on the cingulate gyrus, are the most suitable indications. ${ }^{3}$

SEEG provides a number of advantages over conven- tional methods when addressing cingulate gyrus epilepsy. First, in some cases, the epileptogenic zone involves the cingulate gyrus as well as the cingulate sinus or superior frontal sulcus (e.g., bottom-of-sulcus FCD). Depth electrodes can be designed to penetrate the lateral cortical surface, the bottom-of-sulcus, and the mesial cortical surface at the same time. Second, it is difficult to lateralize based only on the semiology and scalp EEG readings of cingulate gyrus epilepsy. SEEG can be used to perform bilateral analysis simultaneously. Phase II studies can be performed more comprehensively via SEEG. Third, the fact that SEEG is less invasive than subdural electrode implantation is welcomed by patients with epilepsy. Note that when using SEEG, a craniotomy for SDG/strip implantation can replace multiple wounds with minimal invasiveness ( $<3 \mathrm{~mm}$ at each penetration point). Fourth, SEEG can help to identify close relationships with other structures of the limbic system. Fifth, SEEG can help to identify the early spreading area. For instance, the emotional component of the early seizure presentation may be indicative of early spreading to the amygdala. Sixth, SEEG can help to establish surgical margins, particularly for MRI-negative patients or those with FCD type I.

The SEEG schema can be customized to the unique characteristics of cingulate gyrus epilepsy in each patient. Some researchers have reported using SEEG with anterior cingulate gyrus epilepsy, ${ }^{10}$ whereas other researchers have reported epilepsy associated with the posterior cingulate gyrus. ${ }^{9}$ The one commonality is the fact that they simultaneously cover the lateral cortical surface, the bottomof-sulcus, and the mesial cortical surface. Depending on subsequent semiological presentations, the related limbic structures, such as the orbital-frontal gyrus, amygdala, hippocampus, and even the fusiform gyrus, should be covered. 


\section{Using iEEG to Explore the Dynamic Topologies of HFO Networks in Cingulate Gyrus Epilepsy}

The advantage of iEEG when exploring the dynamic topologies lies in the ability to obtain 3D delineations of the hypothetical epileptic network through the use of multiple intracerebral electrodes. Precise stereotactic guidance facilitates the mapping out of relevant cortical structures implicated in seizure generation and early propagation. It also makes it possible to establish the dynamic topologies of cortical and subcortical functional networks.

The quantifiable results provided by iEEG analysis can help to define and elucidate ictogenesis through the extraction of spatial and temporal information from complex signals recorded directly within the epileptic human brain. Time and frequency analysis of iEEG signals can be used to define changes in the relationship between the epileptogenic zone, ictal onset zone, irritative zone, and symptomatic zone during the transition from the interictal to the ictal state. When treating patients with cingulate gyrus epilepsy, it would be highly beneficial to understand the close relationships with other limbic structures and the overlying neocortex. Nonlinear correlation-based or coherence-based studies have revealed preferential interactions occurring between subregions of the limbic system during the generation of epileptic patterns. We determined that early electrodecremental events involving HFO between 80 and $250 \mathrm{~Hz}$ were associated not only with the epileptogenic zone but also bilaterally with the head and body of the hippocampus. This may explain the strong relationship between the cingulate gyrus and the hippocampus in almost all patients with cingulate epilepsy.

\section{Surgical Considerations for Cingulate Gyrus Epilepsy}

Resection is usually performed after chronic invasive EEG recording to identify the seizure onset zone and/or potential epileptogenic zone. Nonetheless, this poses a number of issues that must be considered prior to surgery. Surgeons must determine the means of resection as well as the size of the resection. Resection of any part of the cingulate gyrus poses a risk of transient motor dysfunction. It is well known that the motor cingulate area poses the greatest risk; however, we suggest using IONM during resection. IONM provides important data including motor evoked potentials with D-wave, somatosensory evoked potentials with central sulcus localization, cortical mapping, subcortical mapping, and continuous EEG monitoring to avoid afterdischarges and seizures during mapping. SMA syndrome occurred in $28 \%$ of the patients in this study. Gross motor function is usually recovered within 2 weeks, but fine motor function requires training for at least $1-2$ years.

Note that after identifying the epileptogenic lesion or seizure onset zone, we found that the outcome of resection for mesial extratemporal lobe epilepsy (including cingulate gyrus epilepsy) can be as good as that of temporal lobe epilepsy. ${ }^{19,20}$ In the current study, $67 \%$ of the patients were seizure free and $17 \%$ reported a significant reduction in the intensity of seizures. These findings are similar to those reported by von Lehe et al. in 2012, in which $62 \%$ of the patients were seizure free and $76 \%$ reported a satisfactory outcome in terms of seizure frequency. ${ }^{27}$ In 1 study, the seizure-free rate from posterior cingulate gyrus epilepsy reached $71 \%$ ( $n=5 / 7$ with Engel classification IA or IB). ${ }^{9}$ These results fully justify efforts to locate lesions on the cingulate gyrus.

\section{Study Limitations}

Due to the low incidence rate of cingulate gyrus epilepsy in the general population, the number of patients in this study was small. We recognize the potential for selection bias when a patient group is not large enough. As with all invasive procedures, there are inherent limitations to any iEEG study, regardless of the methodology (e.g., subdural EEG, or SEEG). The limited number of contacts located in the epileptic network could easily lead to sampling error when the network is scaled up. Note also that we condensed the complex network features within several scalar values in an attempt to explain and compare network attributes across the patient population. These simplified tools appear meaningful; however, they may overlook the details of focal network features of individual seizures. Concentrating on these focal characteristics may fail to take into account the overall features of bihemispheric seizure activity that emerges during seizure propagation.

\section{Acknowledgments}

This work was supported in part by the Ministry of Science and Technology, Taiwan, under projects 105-2218-E-009-027, 106-2221-E-009-068, and 107-2314-B-075-059. This work was also supported in part by the Taipei Veterans General Hospitals and University System of Taiwan Joint Research Program (VGHUST107-G1-4-3).

\section{References}

1. Alkawadri R, Mickey BE, Madden CJ, Van Ness PC: Cingulate gyrus epilepsy: clinical and behavioral aspects, with surgical outcomes. Arch Neurol 68:381-385, 2011

2. Alkawadri R, So NK, Van Ness PC, Alexopoulos AV: Cingulate epilepsy: report of 3 electroclinical subtypes with surgical outcomes. JAMA Neurol 70:995-1002, 2013

3. Arya R, Mangano FT, Horn PS, Holland KD, Rose DF, Glauser TA: Adverse events related to extraoperative invasive EEG monitoring with subdural grid electrodes: a systematic review and meta-analysis. Epilepsia 54:828-839, 2013

4. Baleydier C, Mauguiere F: The duality of the cingulate gyrus in monkey. Neuroanatomical study and functional hypothesis. Brain 103:525-554, 1980

5. Bancaud J, Talairach J: Clinical semiology of frontal lobe seizures. Adv Neurol 57:3-58, 1992

6. Beckmann M, Johansen-Berg H, Rushworth MF: Connectivity-based parcellation of human cingulate cortex and its relation to functional specialization. J Neurosci 29:1175-1190, 2009

7. Bonini F, McGonigal A, Trébuchon A, Gavaret M, Bartolomei F, Giusiano B, et al: Frontal lobe seizures: from clinical semiology to localization. Epilepsia 55:264-277, 2014

8. Cossu M, Cardinale F, Castana L, Citterio A, Francione S, Tassi L, et al: Stereoelectroencephalography in the presurgical evaluation of focal epilepsy: a retrospective analysis of 215 procedures. Neurosurgery 57:706-718, 2005

9. Enatsu R, Bulacio J, Nair DR, Bingaman W, Najm I, Gonzalez-Martinez J: Posterior cingulate epilepsy: clinical and neurophysiological analysis. J Neurol Neurosurg Psychiatry 85:44-50, 2014

10. Gonzalez-Martinez J, Bulacio J, Alexopoulos A, Jehi L, 
Bingaman W, Najm I: Stereoelectroencephalography in the "difficult to localize" refractory focal epilepsy: early experience from a North American epilepsy center. Epilepsia 54:323-330, 2013

11. Kramer MA, Eden UT, Cash SS, Kolaczyk ED: Network inference with confidence from multivariate time series. Phys Rev E Stat Nonlin Soft Matter Phys 79:061916, 2009

12. McGovern RA, Ratneswaren T, Smith EH, Russo JF, Jongeling AC, Bateman LM, et al: Investigating the function of deep cortical and subcortical structures using stereotactic electroencephalography: lessons from the anterior cingulate cortex. J Vis Exp 98:e52773, 2015

13. O'Muircheartaigh J, Richardson MP: Epilepsy and the frontal lobes. Cortex 48:144-155, 2012

14. Powell R, Elwes R, Hamandi K, Mullatti N: Cingulate gyrus epilepsy. Pract Neurol 18:447-454, 2018

15. Souirti Z, Landré E, Mellerio C, Devaux B, Chassoux F: Neural network underlying ictal pouting ("chapeau de gendarme") in frontal lobe epilepsy. Epilepsy Behav 37:249257,2014

16. Spencer SS, Goncharova II, Duckrow RB, Novotny EJ, Zaveri HP: Interictal spikes on intracranial recording: behavior, physiology, and implications. Epilepsia 49:1881-1892, 2008

17. Talairach J, Bancaud J, Szikla G, Bonis A, Geier S, Vedrenne $\mathrm{C}$ : [New approach to the neurosurgery of epilepsy. Stereotaxic methodology and therapeutic results. 1. Introduction and history.] Neurochirurgie 20 (Suppl 1):1-240, 1974 (French)

18. Tang H, Yu HY, Chou CC, Crone NE, Madsen JR, Anderson WS, et al: Cascade of neural processing orchestrates cognitive control in human frontal cortex. eLife 5:e12352, 2016

19. Theys T, Decramer T, Van Paesschen W, De Tiège X, Minotti $\mathrm{L}$, von Lehe M, et al: Facing the hidden wall in mesial extratemporal lobe epilepsy. Epileptic Disord 20:1-12, 2018

20. Theys T, Minotti L, Tassi L, Lo Russo G, Benabid AL, Kahane P, et al: Mesial extratemporal lobe epilepsy: clinical features and surgical strategies. Neurosurgery 80:269-278, 2017

21. Vogt BA, Finch DM, Olson CR: Functional heterogeneity in cingulate cortex: the anterior executive and posterior evaluative regions. Cereb Cortex 2:435-443, 1992

22. Vogt BA, Miller MW: Cortical connections between rat cingulate cortex and visual, motor, and postsubicular cortices. J Comp Neurol 216:192-210, 1983

23. Vogt BA, Nimchinsky EA, Vogt LJ, Hof PR: Human cingulate cortex: surface features, flat maps, and cytoarchitecture. J Comp Neurol 359:490-506, 1995
24. Vogt BA, Pandya DN: Cingulate cortex of the rhesus monkey: II. Cortical afferents. J Comp Neurol 262:271-289, 1987

25. Vogt BA, Pandya DN, Rosene DL: Cingulate cortex of the rhesus monkey: I. Cytoarchitecture and thalamic afferents. J Comp Neurol 262:256-270, 1987

26. Vogt BA, Paxinos G: Cytoarchitecture of mouse and rat cingulate cortex with human homologies. Brain Struct Funct 219:185-192, 2014

27. von Lehe M, Wagner J, Wellmer J, Clusmann H, Kral T: Epilepsy surgery of the cingulate gyrus and the frontomesial cortex. Neurosurgery 70:900-910, 2012

28. Whitney R, AlMehmadi S, Go C, Ochi A, Otsubo H, Bradbury L, et al: Spiders, ladybugs and bees: a case of unusual sensations in a child with cingulate epilepsy. Epilepsy Behav Case Rep 8:1-6, 2017

\section{Disclosures}

The authors report no conflict of interest concerning the materials or methods used in this study or the findings specified in this paper.

\section{Author Contributions}

Conception and design: Lee, Chou. Acquisition of data: Lee, Chou, Lin, YH Chen, Yu, C Chen, HH Chen. Analysis and interpretation of data: Lee, Chou, Lin, YH Chen, Peng, Hsiao, Yu, C Chen, HH Chen. Drafting the article: Lee. Critically revising the article: all authors. Reviewed submitted version of manuscript: Chou. Approved the final version of the manuscript on behalf of all authors: Lee. Statistical analysis: Lee, YH Chen, Peng, Hsiao. Administrative/technical/material support: Lee, Chou, Lin, YH Chen, Peng, Hsiao, Yu, C Chen, HH Chen. Study supervision: Chou, Lin, Yu, C Chen, Shih.

\section{Supplemental Information \\ Videos \\ Video 1. https://vimeo.com/389467514.}

\section{Correspondence}

Cheng-Chia Lee: Neurological Institute, Taipei Veterans General Hospital, Taipei, Taiwan. yfnaughty@gmail.com; cclee12@ vghtpe.gov.tw. 